\title{
A new method to determine the thickness of non-edge-on disk galaxies
}

\author{
Y. Zhao, Q. Peng, and T. Hu \\ Department of Astronomy, Nanjing University, Nanjing 210093, PR China \\ e-mail: [yhzhao; qhpeng; taohu] @nju.edu.cn \\ Received 11 July 2005 / Accepted 15 December 2005 \\ ABSTRACT

\begin{abstract}
Aims. We present a new method to determine the thickness of non-edge-on disk galaxies. This method allows us to investigate the mass-to-light ratio of the disk.

Methods. Our method is based on the comparison of observations and theory of the distribution of the vertical velocity dispersion, which is obtained from the solution of three dimensional Poisson equations and the galactic dynamical equation.

Results. As examples, the thickness and mass-to-light ratio of two disk galaxies, NGC 1566 and NGC 5247, which have been of this method. However, due to the small sample size available, the results should be confirmed on other samples of galaxies.
\end{abstract} \\ extensively studied by spectroscopy, have been calculated. The calculated results are consistent with observations and support the use
}

Key words. Galaxy: disk - Galaxy: kinematics and dynamics - Galaxy: structure - galaxies: individual: NGC 1566 galaxies: individual: NGC 5247

\section{Introduction}

The thickness of disk galaxies is a very important parameter to understand these objects. Disk galaxies are very thin relative to the length of the disk. Sancisi \& Allen (1979) estimated the thickness of the edge-on Sb galaxy NGC 891 on the basis of observation of neutral hydrogen. Van der Kruit \& Searle (1981a) proposed a model for light distribution in the disks of edge-on spiral galaxies, assuming that a galaxy has a locally isothermal, self-gravitating, truncated and exponential disk. The model has the feature of being isothermal in the $z$-direction at all radii with a scale parameter of $z_{0}$ and has an exponential dependence of surface brightness on $r$ with a scale length of $r_{\mathrm{d}}$. The space luminosity of this model can be described by

$L(r, z)=L_{0} \mathrm{e}^{-r / r_{\mathrm{d}}} \sec h^{2}\left(z / z_{0}\right)$.

With this model, van der Kruit \& Searle (1981a,b, 1982a,b, KS hereafter) determined $r_{\mathrm{d}}$ and $z_{0}$ for several edge-on galaxies without an appreciable bulge. Unfortunately, this method may not be suitable for general disk galaxies, e.g. face-on galaxies.

Peng (1988) proposed a method to measure the thickness of face-on galaxies on the basis of asymptotic formulae of the disturbed gravitational potential. A revised method based on the exact integral expression for disturbed gravitational potential has been presented by Zhao et al. (2004) to estimate the thickness of face-on disk galaxies. The results obtained with these two correlative methods should be confirmed with the results obtained independently.

In this paper, we present the solution of the Jeans equation along the $z$-direction and then describe how to use this solution to determine the thickness of face-on disk galaxies. In Sect. 3, we show the applications of our method to two near face-on galaxies, NGC 1566 and NGC 5247, which have been extensively studied with spectroscopy. A brief discussion is given in the last section of this paper.

\section{Method}

\subsection{The distribution of the vertical velocity dispersion}

For a stable, axisymmetric galaxy, the Jeans equation along the $z$-direction is

$\frac{\partial}{\partial z}\left(\rho\left\langle V_{z}^{2}\right\rangle\right)=-\rho \frac{\partial \varphi}{\partial z}$

where $\left\langle V_{z}^{2}\right\rangle$ is square of the $z$-direction velocity dispersion.

As the first of the fundamental assumptions, we accept Parenago's density distribution law along the $z$-direction for a finite thickness galaxy,

$\rho(r, z)=\rho(r, 0) \mathrm{e}^{-\alpha|z|}=\frac{\alpha}{2} \sigma(r) \mathrm{e}^{-\alpha|z|}$,

where $\alpha$ is a parameter the reciprocal of which is half of the effective thickness, $H$, of a galaxy. $\alpha$ may be taken as a constant since the scale height is basically independent of the radius at least for the thick disk of our Galaxy (e.g. see Freeman 1987). $\sigma(r)$ is its surface density. de Grijs \& van der Kruit (1996) have confirmed that the simple exponential fit of the vertical stellar light distribution is a good approximation. Hence, the potential caused by this density can be found by solving the Poisson equation of the potential

$\nabla^{2} \varphi(r, z)=2 \pi G \alpha \sigma(r) \mathrm{e}^{-\alpha|z|}$

The rigorous solution of the potential has been given by Peng et al. (1978)

$$
\begin{aligned}
\varphi(r, z)= & -\pi G \alpha \\
& \cdot \int_{0}^{\infty} \frac{2}{\beta^{2}-\alpha^{2}}\left(\beta \mathrm{e}^{-\alpha|z|}-\alpha \mathrm{e}^{-\beta|z|}\right) J_{0}(\beta r) s(\beta) \mathrm{d} \beta,
\end{aligned}
$$


Table 1. Parameters of NGC 1566 and NGC 5247.

\begin{tabular}{cccccc}
\hline \hline NGC & Inclination & Classification & Distance $^{a}(\mathrm{Mpc})$ & $M_{\mathrm{H}}(\mathrm{mag})$ & Ref. \\
\hline 1566 & $28^{\circ} \pm 5^{\circ}$ & Sc(s)I, Seyfert 1 & 17.4 & -24.0 & $(1),(2),(4)$ \\
5247 & $20^{\circ} \pm 3^{\circ}$ & Sc(s)I-II & 16.0 & -23.2 & $(1),(3),(4)$ \\
\hline
\end{tabular}

${ }^{a}$ Obtained based on $H_{0}=75 \mathrm{~km} \mathrm{~s}^{-1} \mathrm{Mpc}^{-1}$.

References: (1) Sandage \& Tammann (1981); (2) Bottema (1992); (3) van der Kruit \& Freeman (1986); (4) Jarrett et al. (2003).

where

$s(\beta)=\int_{0}^{\infty} r J_{0}(\beta r) \sigma(r) \mathrm{d} r$.

$J_{0}(\beta r)$ is the well known Bessel function of order 0 .

Then using Eq. (5), we integrate Eq. (2) from $z$ to $\infty(z \geq 0)$ at both sides of the equal sign. It follows that (Huang et al. 1979)

$\left(\rho\left\langle V_{z}^{2}\right\rangle\right)_{z \geq 0}=-2 \pi G \alpha^{2} \rho(r, 0)$

$$
\int_{0}^{\infty} \frac{\beta}{\beta^{2}-\alpha^{2}}\left[\frac{1}{\alpha+\beta} \mathrm{e}^{-(\alpha+\beta) z}-\frac{1}{2 \alpha} \mathrm{e}^{-2 \alpha z}\right] J_{0}(\beta r) s(\beta) \mathrm{d} \beta .
$$

Hence, on the galactic plane $(z=0),\left\langle V_{z}^{2}\right\rangle$ can be written as

$$
\begin{aligned}
\left\langle V_{z}^{2}\right\rangle_{(z=0)} & =\pi G \alpha \int_{0}^{\infty} \frac{\beta}{(\alpha+\beta)^{2}} J_{0}(\beta r) s(\beta) \mathrm{d} \beta \\
& =\frac{\pi G}{\alpha} \sigma(r)[1-P(r)]
\end{aligned}
$$

where

$$
\begin{aligned}
& P(r, \alpha)=\frac{1}{\sigma(r)} \int_{0}^{\infty} \beta \zeta(\alpha, \beta) J_{0}(\beta r) s(\beta) \mathrm{d} \beta, \\
& \zeta(\alpha, \beta)=1-\frac{1}{(1+\beta / \alpha)^{2}} .
\end{aligned}
$$

\subsection{The thickness parameter $\alpha$}

Freeman (1970) studied 36 spiral and S0 galaxies with surface photometry and assuming that a galaxy has an infinitesimally thin disk, he showed that the radial light distribution in the disks of spiral galaxies can be described by an exponentially decreasing surface-brightness with increasing galactocentric radius. Along the vertical direction, de Grijs \& van der Kruit (1996) showed that simple exponential fits are good approximations of the stellar light distribution. Thus, the model to account for the space-luminosity can be described by

$L(r, z)=L_{0} \mathrm{e}^{-r / r_{d}} \mathrm{e}^{-\alpha|z|}$,

where $r$ is the position along the major axis, $L_{0}$ the central space luminosity in the plane of the galaxy, $r_{\mathrm{d}}$ the scale length of the disk. From this we calculate the face-on distribution of surface-brightness

$I(r)=I_{0} \mathrm{e}^{-r / r_{\mathrm{d}}}$,

where $I_{0}$ is the central surface brightness of the disk.

Making use of the observed constant color index (van der Kruit \& Searle 1981b, 1982a), van der Kruit \& Freeman (1986) pointed out that the mass-to-light ratio of the old disk, $\Upsilon_{*}=(M / L)_{\text {old disk }}$, is approximately constant with the radius. The projected surface density is

$\sigma(r)=\Upsilon_{*} I(r)=\Upsilon_{*} I_{0} \mathrm{e}^{-r / r_{\mathrm{d}}}=\sigma_{0} \mathrm{e}^{-r / r_{\mathrm{d}}}$,

where $\sigma_{0}$ is the surface density at $r=0$.

Therefore, Eq. (6) could be reduced by substituting $\sigma(r)$ with Eq. (12):

$s(\beta)=\int_{0}^{\infty} r J_{0}(\beta r) \sigma(r) \mathrm{d} r=\Upsilon_{*} I_{0} \frac{r_{\mathrm{d}}^{2}}{\left(1+r_{\mathrm{d}}^{2} \beta^{2}\right)^{3 / 2}}$.

$\left\langle V_{z}^{2}\right\rangle$ may be rewritten as

$$
\begin{aligned}
\left\langle V_{z}^{2}\right\rangle & =\frac{\pi G}{\alpha} \Upsilon_{*} I(r)[1-P(r)] \\
& =\frac{\pi G}{\alpha} \Upsilon_{*} I_{0}\left[\mathrm{e}^{-r / r_{\mathrm{d}}}-P_{1}(r)\right],
\end{aligned}
$$

where

$$
\begin{aligned}
P_{1}(r) & =P(r) \mathrm{e}^{-r / r_{\mathrm{d}}} \\
& =\int_{0}^{\infty} \frac{r_{\mathrm{d}}^{2}}{\left(1+r_{\mathrm{d}}^{2} \beta^{2}\right)^{3 / 2}} \beta\left[1-\frac{1}{(1+\beta / \alpha)^{2}}\right] J_{0}(\beta r) \mathrm{d} \beta .
\end{aligned}
$$

Hence, if we know the $I_{0}, r_{\mathrm{d}}$ from photometric studies and $\left\langle V_{z}^{2}\right\rangle$ from spectroscopic observations, we can calculate the thickness parameter $\alpha$ with Eq. (14) and therefore obtain the thickness of the galaxy.

\section{Application}

We found extensive spectroscopic studies of two nearly face-on galaxies, NGC 1556 and NGC 5247. We use these two galaxies as examples of our method. General information on these two galaxies are summarized in Table 1.

\subsection{Photometric decomposition}

Although there are photometric results for NGC 1566 (de Vaucouleurs 1973; Bottema 1992) and 5247 (van der Kruit \& Freeman 1986), we cannot use these data directly since the photometric decomposition is very important for our purpose. The photometric decomposition can disentangle the contributions to the total luminosity of the bulge and disk, and this will allow the identification of the disk region. Moreover, because the infrared images suffer much less extinctions than optical ones do, we use the 2MASS $H$-band images to obtain photometric measurements.

To obtain photometric parameters, $r_{\mathrm{d}}$ and $\mu_{0}$, we use the two dimensional image-fitting algorithm GALFIT (Peng et al. 2002) designed to extract structural parameters directly from the galaxy images. GALFIT assumes a two-dimensional model profile for the galaxy. The functional forms of the models we choose to fit include combinations of a Gaussian, a Sérsic $r^{1 / n}$ law and 
Table 2. Bulge-disk decompositions for NGC 1566 and NGC 5247. The fitting functions are a Gaussian (for the nucleus), Sérsic $r^{1 / n}$ law (for the bulge) and an exponent (for the disk).

\begin{tabular}{|c|c|c|c|c|c|c|c|c|}
\hline Component & $\begin{array}{c}M_{\mathrm{tot}} \\
(\mathrm{mag})\end{array}$ & $\begin{array}{c}F W H M / r_{\mathrm{e}} / r_{\mathrm{d}}{ }^{a} \\
(\mathrm{kpc})\end{array}$ & $\begin{array}{c}\mu_{\mathrm{e}} / \mu_{0}{ }^{b} \\
\left(\mathrm{mag} \operatorname{arcsec}^{-2}\right)\end{array}$ & $\begin{array}{r}\text { PA } \\
(\circ) \\
\end{array}$ & $n$ & $q$ & $c$ & $\chi_{v}^{2}$ \\
\hline \multicolumn{9}{|c|}{ NGC 1566} \\
\hline Nucleus & $11.70 \pm 0.03$ & $0.32 \pm 0.01$ & & $88.02 \pm 2.36$ & & $0.89 \pm 0.01$ & $0.26 \pm 0.11$ & \\
\hline Bulge & $9.25 \pm 0.02$ & $0.92 \pm 0.03$ & $17.05 \pm 0.06$ & $12.21 \pm 0.48$ & $1.65 \pm 0.03$ & $0.79 \pm 0.00$ & $-0.28 \pm 0.01$ & \\
\hline Disk & $7.65 \pm 0.00$ & $3.29 \pm 0.04$ & $17.20 \pm 0.04$ & $-15.62 \pm 0.32$ & $\ldots$ & $0.73 \pm 0.00$ & $-0.29 \pm 0.02$ & 0.310 \\
\hline \multicolumn{9}{|c|}{ NGC 5247 } \\
\hline Bulge & $10.69 \pm 0.01$ & $0.67 \pm 0.02$ & $18.06 \pm 0.04$ & $42.20 \pm 1.44$ & $1.24 \pm 0.01$ & $0.87 \pm 0.00$ & $0.01 \pm 0.04$ & \\
\hline Disk & $7.84 \pm 0.00$ & $4.78 \pm 0.03$ & $18.60 \pm 0.03$ & $-18.72 \pm 0.70$ & $\ldots$ & $0.80 \pm 0.00$ & $-0.08 \pm 0.02$ & 0.130 \\
\hline
\end{tabular}

${ }^{a}$ For the nucleus it is $F W H M$, for the bulge it is $r_{\mathrm{e}}$, and for the disk it is $r_{\mathrm{d}} ;{ }^{b}$ for the bulge it is $\mu_{\mathrm{e}}$, and for the disk it is $\mu_{0}$.
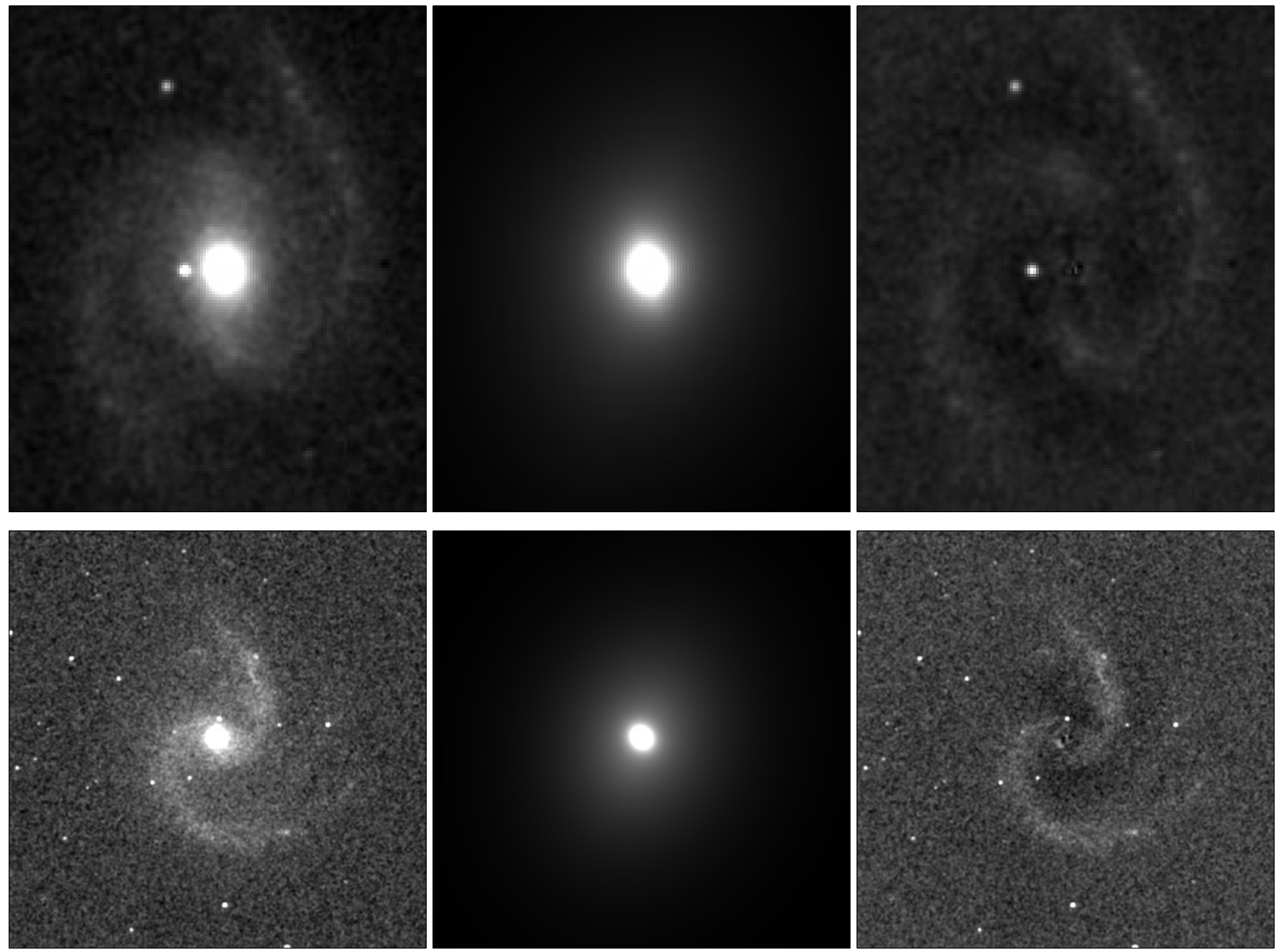

Fig. 1. For both galaxies we show the original images (left), the smooth and symmetric model images fitted by GALFIT (middle) and the residual images (right). For NGC 1566 (top), the size of each field is $2 ! 4 \times 2$ '.9; for NGC 5247 (bottom), the size is 6!7 × 6!7.

an exponential disk profile. For the case of a Seyfert 1 galaxy, NGC 1566, we use all three functions to fit, while we use the last two functions for NGC 5247. We fit the following: the ( $x$, $y)$ position of the center, $M_{\text {tot }}$ (the total magnitude of the component), $r_{\mathrm{e}}$ (the effective radius), $n$ (the Sérsic index), $q$ (the axis ratio), $r_{\mathrm{d}}$ (the scale length of the exponential disk), the major position angle (PA), and $c$ (the diskiness/boxiness index). The detailed parameters of all fitted components are listed in Table 2. The original images, the smooth and symmetric model images fitted by GALFIT and the residual images are shown in the left, middle and right panels in Fig. 1.
To check whether the fitted results are reliable, we examine the residual images obtained by subtracting the fitted models. In both cases we found no obvious evidence (except for the expectant spiral arms) for components beyond the fitted models. Furthermore, we also measure surface brightness profiles using the $I R A F^{1} / E L L I P S E$ algorithm. In Fig. 2 we show the

1 IRAF is distributed by the National Optical Astronomy Observatories, which is operated by Associated of Universities for Research in Astronomy, Inc., under cooperative agreement with the National Science Foundation. 


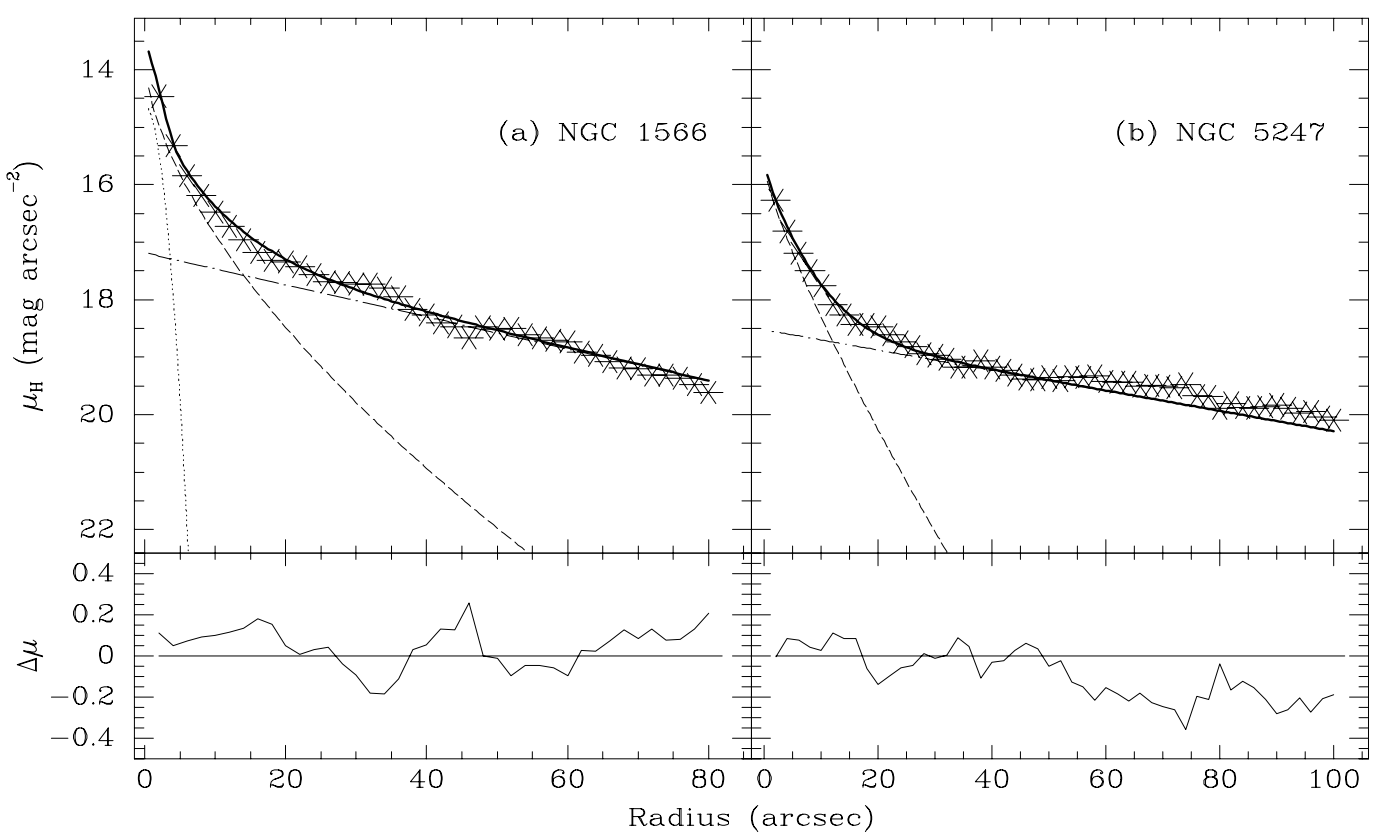

Fig. 2. 2MASS $H$-band radial surface brightness profiles. Symbols show the radial profiles obtained from ELLIPSE, the dotted line shows the Seyfert core component fitted to the Gaussian function, the dashed lines show the bulge components fitted to the Sérsic $r^{1 / n}$ law, and the dasheddotted lines show the disk components fitted to the exponential law. The thick solid lines are the superposition of all the components. The lower portion of each panel shows the difference between the data (ELLIPSE) and the fits (GALFIT).

radial profiles obtained from the isophote fitting by ELLIPSE and the profiles of the chosen model for each galaxy. The differences between the observational data (ELLIPSE) and the models (GALFIT) range mostly over $\pm 0.2 \mathrm{mag} \operatorname{arcsec}^{-2}$, except for the regions distorted by the spiral arms at large radii. Thus, we conclude that our global fits are reliable.

\subsection{Fitting the observed dispersion data}

The vertical velocity dispersions of NGC 1566 were obtained from Table 4, Col. 4 in Bottema (1992); NGC 5247 from Table 1, Col. 4 in van der Kruit \& Freeman (1986). As these two galaxies are not exactly face-on (see Table 1), this will affect the observational data in two ways: (1) the observed velocity dispersion is not exactly equal to the vertical velocity dispersion. The observed dispersion is approximately $4 \%$ higher than the real vertical dispersion for NGC 1566 (Bottema 1992), 5\% larger for NGC 5247 (van der Kruit \& Freeman 1986). (2) the observed surface brightness only amounts to about $\cos i$ times the face-on brightness. Before using the photometric and spectroscopic data for fitting, we have corrected for inclination. We present a further discussion about how the inclination affects the fitted results below.

To obtain the thickness parameter $\alpha$, we use a least-squares method to fit the observational data shown in Figs. 3, and 4, for NGC 1566 and NGC 5247 for only points with radius larger than $\sim 2-3 r_{\mathrm{e}}$, where the bulge's contribution to the velocity dispersion may be neglect according to Terzić \& Graham (2005) (see Figs. 11 and 12 in their paper). In the process of fitting, we first adopt $\Upsilon_{*, H}$ (the mass-to-light ratio for the $H$-band) as an empirical value, following Devereux et al. (1987), Oliva et al. (1995) and Giovanardi \& Hunt (1996), and then use Eq. (14) to derive the thickness parameter $\alpha$ and $\chi_{v}^{2}$. We adopt this fitted $\alpha$ to derive the mass-to-light ratio $\Upsilon_{*, H}$. Such iterations are repeated until $\Delta \chi_{v}^{2} / \chi_{v}^{2}\left(\Delta \chi_{v}^{2}\right.$ is the difference between the $\chi_{v}^{2}$ given by two successive fitting) is less than several times $10^{-4}$.

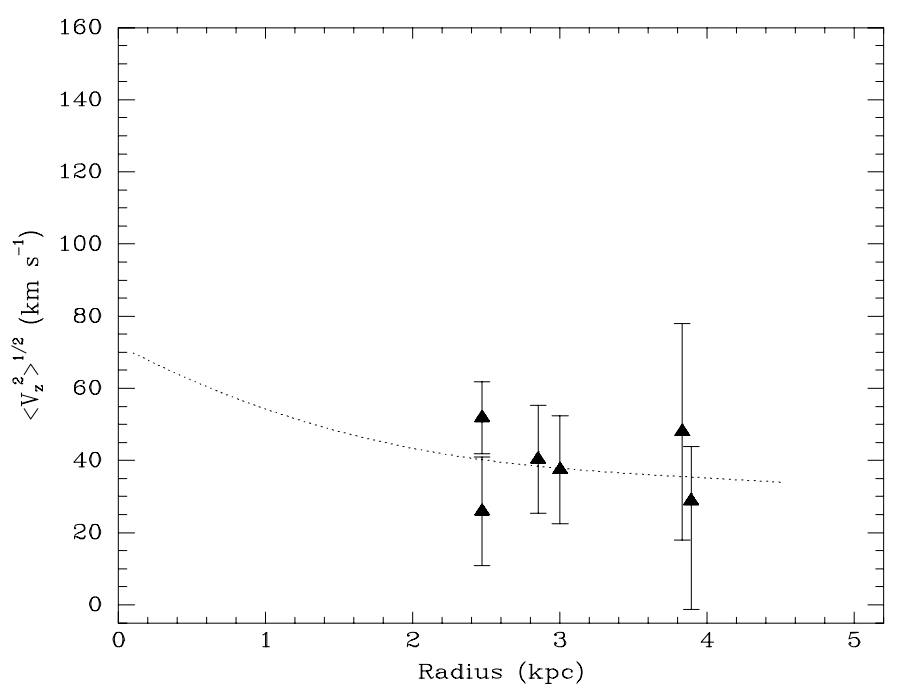

Fig. 3. Observed (filled triangles) and fitted (dotted line) distribution of vertical stellar velocity dispersion of the disk in NGC 1566. The observed data are from Bottema (1992), but the data which belong to the Seyfert core and the bulge are not included in the figure, and not taken into account for the determination of the thickness parameter $\alpha$. The line is our least-squares fitted results from Eq. (14) in the text.

The parameters needed in our fitting process and the fitted results are listed in Table 3. The best fitted results are also shown in Figs. 3 and 4 with dotted lines.

The values of $\chi_{v}^{2}$ given by our least-squares fitting for NGC 1566 and NGC 5247 are 0.48 and 0.22 , respectively (see Table 3). These values of $\chi_{v}^{2}$ are somewhat smaller than statistically acceptable, presumably because of the large measurement errors of the observed data and the small samples used for the fits. The best-fit results with our model are given in Figs. 3 and 4. They show that our method may be realistic and could be used 
Table 3. Photometric parameters and least-square fitted results for NGC 1566 and NGC 5247.

\begin{tabular}{ccccccc}
\hline \hline NGC & $\begin{array}{c}I_{0} \\
\left(L_{\odot} \mathrm{pc}^{-2}\right)\end{array}$ & $\begin{array}{c}\Upsilon_{*} \\
\left(M_{\odot} / L_{\odot}\right)\end{array}$ & $\chi_{v}^{2}$ & $\begin{array}{c}\alpha \\
\left(\mathrm{kpc}^{-1}\right)\end{array}$ & $\begin{array}{c}H^{a} \\
(\mathrm{kpc})\end{array}$ & Band \\
\hline 1566 & $1193.6 \pm 29.7$ & $0.49 \pm 0.03$ & 0.48 & $1.75 \pm 0.56$ & $1.14 \pm 0.36$ & $H$ \\
5247 & $328.7 \pm 4.5$ & $0.91 \pm 0.03$ & 0.22 & $1.32 \pm 0.52$ & $1.52 \pm 0.60$ & $H$ \\
\hline
\end{tabular}

${ }^{a}$ Calculated according to $\mathrm{H}=2 / \alpha$.

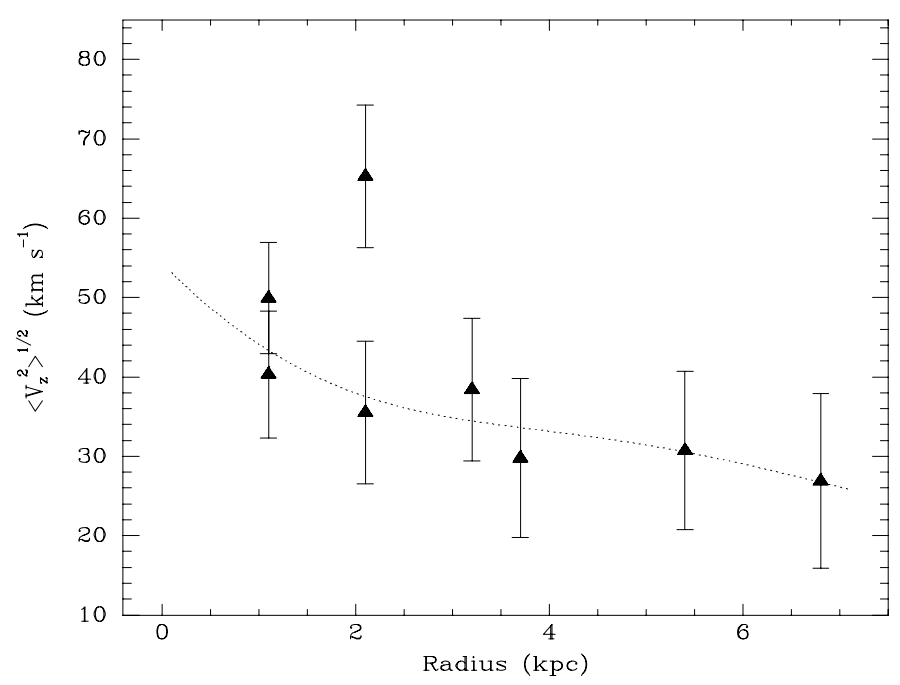

Fig. 4. Observed (filled triangles) distribution and our fitted results (dotted line) with $H$-band photometric data for the disk of NGC 5247. The observations are obtained by van der Kruit \& Freeman (1986). The data belonging to the bulge are excluded from this figure and are not taken into account for our fit.

widely to measure the thicknesses of non-edge-on disk galaxies. Also, our method can derive the mass-to-light ratio simultaneously. However, the values obtained are overestimated since the mass contribution of the dark matter halo is neglected, which is a reasonable assumption. The kinematic data cover a radial range where luminous matter can be assumed to dominate the galaxy dynamics. As shown in Table 3, the derived mass-to-light ratio for these two galaxies are consistent with the empirical values (Devereux et al. 1987; Oliva et al. 1995; Giovanardi \& Hunt 1996). This also suggests that our method is reasonable.

If we only need to know the vertical velocity dispersion at some exact radius (must be larger than $\sim 2 r_{\mathrm{e}}$ ),$\left\langle V_{z, r_{1}}^{2}\right\rangle^{1 / 2}$, and the photometric results, e.g. the scale length of the disk $r_{\mathrm{d}}$ and the central extrapolated surface brightness $I_{0}$, then, Eq. (14) can be rewritten as

$$
\left\langle V_{z, r_{1}}^{2}\right\rangle \alpha+\pi G \Upsilon_{*} I_{0} P_{1}\left(r_{1}, \alpha\right)-\pi G \Upsilon_{*} I_{0} \mathrm{e}^{-r_{1} / r_{\mathrm{d}}}=0 .
$$

The thickness parameter, $\alpha$, is the only unknown parameter in this equation if we have assumed the mass-to-light ratio. Alternatively, we can use the surface density-weighted average velocity dispersion, $\overline{\left\langle V_{z}^{2}\right\rangle}$, to estimate the thickness parameter $\alpha$ through the following equation,

$\overline{\left\langle V_{z}^{2}\right\rangle}=\frac{\int_{0}^{r_{\mathrm{d}}} \frac{\pi G}{\alpha} \sigma^{2}(r)[1-P(r)] r \mathrm{~d} r}{\int_{0}^{r_{\mathrm{d}}} \sigma(r) r \mathrm{~d} r}$.

When Eq. (17) is used for measurements, Eq. (12) could be substituted for $\sigma(r)$.

\section{Discussion}

We have proposed a method to determine the thickness of nonedge on disk galaxies $H$, based on photometric results, the scale length of disk $\left(r_{\mathrm{d}}\right)$ and extrapolated central surface brightness $\left(I_{0}\right)$; and spectroscopic result, the vertical stellar velocity dispersion $\left(\left\langle V_{z}^{2}\right\rangle\right)$. The fundamental assumption required is that the disk mass profile is exponential, both along the vertical direction (i.e., $z$-direction, see Eq. (3)), which is different from the isothermal model used by $\mathrm{KS}$, and along the radial direction (i.e., $r$-direction, see Eq. (1)). It has been shown by de Grijs \& van der Kruit (1996) that the best fitting vertical model is more peaked than expected for an isothermal sheet distribution and the simple exponential fits turned out to be good approximations of the vertical surface brightness profile. Peletier \& de Grijs (1997) also find that the $K$-band light (often assumed to trace mass) indeed has a very peaked, almost exponential, vertical distribution in edge-on spirals. In contrast, Gould et al. (1996) find that the vertical distribution of Galactic $M$ dwarfs (which probably trace the stellar disk) is somewhat less peaked.

Because our method is intended for a pure disk galaxy, the photometric decomposition is another important ingredient affecting the fitted result. As described in Sect. 3.1, photometric decomposition allows us to identify the real disk region and the range that the bulge/nucleus (if the galaxy has) affects. We also use the data just outside the effective radius $r_{\mathrm{e}}$ to fit $\alpha$ for NGC 1566, and we obtain a much larger $\chi^{2}$ of 1.8 and a smaller $\alpha$ of $0.96 \mathrm{kpc}^{-1}$. For NGC 5247, we use the data out to the center as there are no data points near the effective radius. This also gives a larger $\chi^{2}$ of 0.35 and a smaller $\alpha$ of $1.0 \mathrm{kpc}^{-1}$. This suggests that we need to select the radial range according to the total mass of the bulge/nucleus. The inclination is another ingredient affecting the fitting results, particularly true for the mass-to-light ratio. Applied corrections for different inclinations will result in different mass-to-light ratio, but almost the same thickness parameter. For the same velocity dispersion data, a smaller inclination gives a larger mass-to-light ratio.

The parameter $H$ should be regarded as about twice the scale height of the best fit of an exponential to the actual distribution. We compared some relevant results, e.g. $r_{\mathrm{d}} / H$ (the scale length to the effective height ratio), $H$ (the effective height) of these three galaxies with those of eight edge-on spirals of KS, directly measured by fitting the photometric data. As shown in Table 4, our results are consistent with KS. This suggests that our new method for determining the thickness of non-edge-on disk galaxies is reasonable and feasible. In Fig. 5 we plot the thickness versus the Hubble type. The continuous line is the fitted result for the crosses, which represent the median value of the thickness for each type. The trend that the thickness of disks decreases along the Hubble sequence is also found by Ma (2002) and Zhao et al. (2004). However, further study of this correlation is needed because the sample used here is small.

The method presented in this paper is difficult to use for measurements of large galactic samples because the distributions of the vertical stellar velocity dispersions are difficult to obtain in 
Table 4. Parameters of the near face-on galaxies from our study compared with those of edge-on galaxies from the study of van der Kruit \& Searle (1981a,b, 1982a,b).

\begin{tabular}{lcccccc}
\hline \hline NGC & RC3 Type & $M_{H}{ }^{a}$ & $r_{\mathrm{d}}{ }^{b}(\mathrm{kpc})$ & $H^{c}(\mathrm{kpc})$ & $r_{\mathrm{d}} / H$ & Ref. \\
\hline 1566 & Sbc & -24.0 & 3.3 & 1.1 & 3.0 & our study \\
5247 & $\mathrm{Sbc}$ & -23.2 & 4.8 & 1.5 & 3.2 & our study \\
891 & $\mathrm{Sb}$ & -23.3 & 4.9 & 0.99 & 5.0 & $(1)$ \\
4013 & $\mathrm{Sb}$ & -22.4 & 3.4 & 1.1 & 3.1 & $(2)$ \\
4217 & $\mathrm{Sb}$ & -22.9 & 3.5 & 1.7 & 2.1 & $(2)$ \\
4244 & $\mathrm{Scd}$ & -20.7 & 2.6 & 0.58 & 4.5 & $(3)$ \\
4565 & $\mathrm{Sb}$ & -23.7 & 5.5 & 0.79 & 7.0 & $(3)$ \\
5023 & $\mathrm{Scd}$ & -19.5 & 2.0 & 0.46 & 4.3 & $(2)$ \\
5907 & $\mathrm{Sc}$ & -23.1 & 5.7 & 0.83 & 6.9 & $(2)$ \\
7814 & $\mathrm{Sab}$ & -23.5 & 8.4 & 2.0 & 4.2 & $(4)$ \\
\hline
\end{tabular}

${ }^{a}$ The magnitudes are all from Jarrett et al. (2003); ${ }^{b} r_{\mathrm{d}}$ in KS is represented by $h ;{ }^{c} H$ is represented by $z_{0}$, which is obtained by fitting the light distribution with $\mu(R, z)=\mu(0,0)(R / h) K_{1}(R / h) \operatorname{sech}^{2}\left(z / z_{0}\right), K_{1}$ is the modified Bessel function, in KS.

References: (1) van der Kruit \& Searle (1981b); (2) van der Kruit \& Searle (1982a); (3) van der Kruit \& Searle (1981a); (4) van der Kruit \& Searle (1982b).

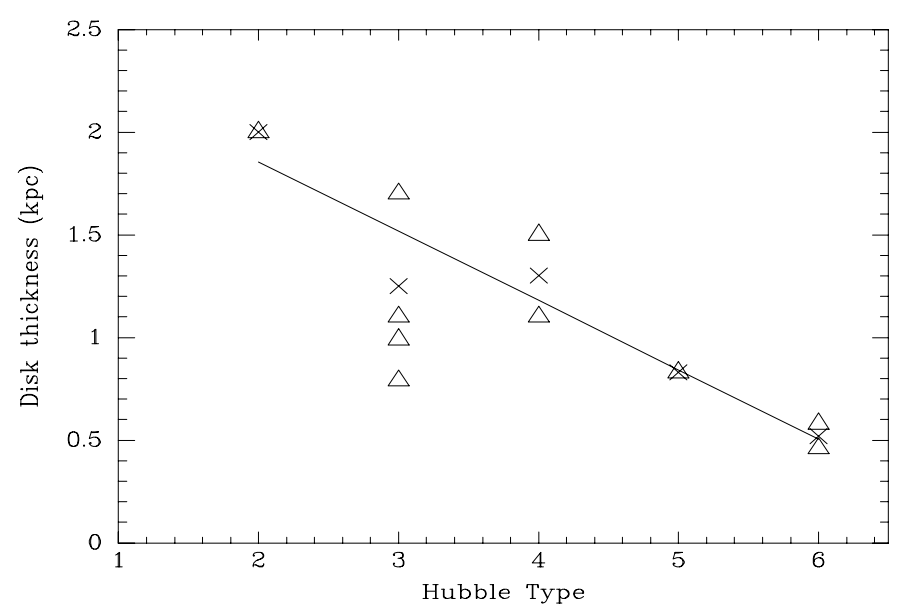

Fig. 5. Thickness plotted versus Hubble type (1, 2, 3, 4, 5 and 6 for Sa, $\mathrm{Sab}, \mathrm{Sb}, \mathrm{Sbc}, \mathrm{Sc}$ and $\mathrm{Scd})$. The continuous line is the fitted result of the median value of each type (represented by the crosses).

practice. The purpose of this work is to test the method proposed by Zhao et al. (2004), which is on the basis of the density wave theory. Thus, we measured the thickness of the disks of NGC 1566 and NGC 5247 by Zhao et al. (2004) and obtained $0.6 \pm 0.4 \mathrm{kpc}$ and $0.7 \pm 0.5 \mathrm{kpc}$, for NGC 1566 and NGC 5247 . These results are somewhat smaller than the results obtained using our method. However, these values are also in the range of the measurement error. Therefore, the method presented in Zhao et al. (2004), which is more easily carried out, might be used to measure the thickness of the disks of nearly face-on galaxies for large samples.

Acknowledgements. The authors are very grateful to Dr. E. M. Corsini for his thoughtful and instructive comments. We are grateful to Dr. Xinlian Luo and Dr. Qiusheng Gu of the Department of Astronomy, Nanjing University for valuable discussions and suggestions. We also thank Dr. T. H. Jarrett. We express our appreciation for the support by the Chinese National Science Foundation No. 10573011, 10273006 and the Doctoral Program Foundation of State Education Commission of China. This paper uses data products from 2MASS, a joint project of the University of Massachusetts and the IPAC/CalTech, funded by NASA and NSF.

\section{Appendix A: Mathematical basis}

To study the effect of finite thickness of the disk on the dynamical properties of disk galaxies, it is convenient to adopt cylindrical coordinates $(r, \theta, z)$ with the galactic center chosen at the origin of the coordinate system. The galactic plane is depicted by $z=0$ and $\theta$ represents the azimuthal coordinate. For disk galaxies with zero thickness, the self-gravitational potential $\Phi$ is governed by Poisson equation

$\nabla^{2} \Phi=4 \pi G \sigma(r, \theta) \delta(z)$

where $G$ is the gravitational constant, $\nabla^{2}$ denotes the Laplacian operator, $\delta(z)$ is Dirac's delta function that confines all the disk mass to the galactic plane and $\sigma(r, \theta)$ represents the surface density. In regions outside the galactic plane $z \neq 0$, Eq. (A.1) then becomes

$\nabla^{2} \Phi(r, \theta, z)=0$

since there is no distribution of matter outside the disk plane. We introduce the Laplace transform for the vertical coordinate $z$ and the Fourier transform for the azimuthal angle $\theta$, namely

$\Phi(r, \theta, z)=\mathrm{e}^{-\mathrm{i} m \theta} \int_{0}^{\infty} U_{\beta}(r) \mathrm{e}^{-\beta|z|} \mathrm{d} \beta$.

Applying these transformations to Eq. (A.2) we then have

$x \frac{\mathrm{d}}{\mathrm{d} x}\left(x \frac{\mathrm{d} U_{\beta}(x)}{\mathrm{d} x}\right)+\left(x^{2}-m^{2}\right) U_{\beta}(x)=0$,

where $x=\beta r$, and the solution of Eq. (A.4) is the well known Bessel function of order $m$

$U_{\beta}(x) \equiv U(\beta r)=-J_{m}(\beta r)$.

Using Eq. (A.5) in Eq. (A.3), we obtain

$\Phi(r, \theta, z)=\mathrm{e}^{-\mathrm{i} m \theta} \int_{0}^{\infty}\left[-J_{m}(\beta r)\right] \mathrm{e}^{-\beta|z|} \mathrm{d} \beta$.

On the other hand, we can integrate Poisson's equation (Eq. (A.1)) with respect to $z$ to obtain the following expression for the surface density $\sigma(r, \theta)$

$\sigma(r, \theta)=\frac{1}{4 \pi G}\left\{\left[\frac{\partial \Phi}{\partial z}\right]_{0^{+}}-\left[\frac{\partial \Phi}{\partial z}\right]_{0^{-}}\right\}$.

Substituting Eq. (A.6) in Eqs. (A.1) and (A.7) we can recast the Poisson equation for disk galaxies with zero thickness in the form

$$
\begin{array}{r}
\nabla^{2}\left\{\int_{0}^{\infty} \mathrm{e}^{-\mathrm{i} m \theta}\left[-J_{m}(\beta r)\right] \mathrm{e}^{-\beta|z|} \mathrm{d} \beta\right\}= \\
\int_{0}^{\infty} \mathrm{e}^{-\mathrm{i} m \theta} 2 \beta J_{m}(\beta r) \delta(z) \mathrm{d} \beta,
\end{array}
$$

$\nabla^{2}\left[J_{m}(\beta r) \mathrm{e}^{-\mathrm{i} m \theta-\beta\left|z-z^{\prime}\right|}\right]=-2 \beta J_{m}(\beta r) \mathrm{e}^{-\mathrm{i} m \theta} \delta\left(z-z^{\prime}\right)$.

\section{Appendix B: Solution of Poisson's equation for an axisymmetric disk}

On the basis of the mathematical treatment just depicted together with the standard method of Green functions, it is straightforward to derive the appropriate Poisson equation for the three-dimensional disk galaxies with finite thickness

$\nabla^{2} \varphi(r, z)=4 \pi G \rho(r, z)$ 
where the vertical distribution of matter is depicted by Parenako's law, $\rho(r, z)=\frac{\alpha}{2} \sigma(r) \mathrm{e}^{-\alpha|z|}$ (see Eq. (3) in the text). To proceed, we apply the Bessel-Fourier transform to $\sigma(r)$ to obtain

$\sigma(r)=\int_{0}^{\infty} \beta J_{0}(\beta r) S(\beta) \mathrm{d} \beta$,

$S(\beta)=\int_{0}^{\infty} r J_{0}(\beta r) \sigma(r) \mathrm{d} r$

where $S(\beta)$ is the Bessel-Fourier transform for $\sigma(r)$. Substituting Eqs. (A.1) and (B.2) into Eq. (B.1), the three-dimensional Poisson equation can be rewritten as

$$
\begin{aligned}
\nabla^{2} \varphi(r, z)= & 2 \pi G \alpha \\
& \cdot \int_{-\infty}^{\infty} \mathrm{e}^{-\alpha\left|z^{\prime}\right|} \mathrm{d} z^{\prime} \int_{0}^{\infty} \beta J_{0}(r \beta) S(\beta) \delta\left(z-z^{\prime}\right) \mathrm{d} \beta .
\end{aligned}
$$

Comparing Eq. (B.4) with Eq. (A.9) we find the formal solution of the gravitational potential for a galactic disk with scale height $H=2 / \alpha$

$\varphi(r, z)=-2 \pi G \alpha \int_{0}^{\infty} J_{0}(r \beta) S(\beta) F(\alpha, \beta, z) \mathrm{d} \beta$,

where

$F(\alpha, \beta, z)=\frac{1}{\beta^{2}-\alpha^{2}}\left[\beta \mathrm{e}^{-\alpha|z|}-\alpha \mathrm{e}^{-\beta|z|}\right]$.

\section{References}

Bottema, R. 1992, A\&A, 257, 69

de Grijs, R., \& van der Kruit, P. C. 1996, A\&AS, 117, 19

de Vaucouleurs, G. 1973, ApJ, 181, 31

Devereux, N. A., Becklin, E. E., \& Scoville, N. 1987, ApJ, 312, 529

Freeman, K. C. 1970 , ApJ, 160,811

Freeman, K. C. 1987, ARA\&A, 25, 603

Giovanardi, C., \& Hunt, L. K. 1996, AJ, 111, 1086

Gould, A., Bahcall, J. N., \& Flynn, C. 1996, ApJ, 465, 759

Jarrett, T. H., Chester, T., Cutri, R., Schneider, S. E., \& Huchra, J. P. 2003, AJ, 125,525

Huang, K. L., Huang, J. H., \& Peng, Q. H. 1978, Acta Astron. Sin., 20, 232

Ma, J. 2002, A\&A, 388, 389

Oliva, E., Origlia, L., Kotilainen, J. K., \& Moorwood, A. F. M. 1995, A\&A, 301, 550

Peletier, R. F., \& de Grijs, R. 1997, Extragalactic Astronomy in the Infared (Edition: Frontières)

Peng, Q. H. 1988, A\&A, 206, 18

Peng, Q. H., Huang, K. L., Huang, J. H., Li, X. Q., \& Su, H. J. 1979, Acta Astron. Sin., 19, 182

Peng, C. Y., Ho, L. C., Impey, C. D., \& Rix, H. 2002, AJ, 124, 266

Sancsi, R., \& Allen, R. T. 1979, A\&A, 74, 73

Sandage, A., \& Tammann, G. A. 1981, A Revised Shapley-Ames Catalog of Bright Galaxies (Washington: Carnegie Institute)

Terzić, B., \& Graham, A. W. 2005 [arXiv: astro-ph/0506192]

van der Kruit, P. C., \& Searle, L. 1981a, A\&A, 95, 105

van der Kruit, P. C., \& Searle, L. 1981b, A\&A, 95, 116

van der Kruit, P. C., \& Searle, L. 1982a, A\&A, 110, 61

van der Kruit, P. C., \& Searle, L. 1982b, A\&A, 110, 79

van der Kruit, P. C., \& Freeman, K. C. 1986, ApJ, 303, 556

Zhao, Y. H., Peng, Q. H., \& Wang, L. 2004, Chin. J. Astron. Astrophs., 4, 51 